\title{
Position error in profiles retrieved from MIPAS observations with a 1-D algorithm
}

\author{
M. Carlotti ${ }^{1}$, E. Arnone ${ }^{2}$, E. Castelli ${ }^{2}$, B. M. Dinelli ${ }^{2}$, and E. Papandrea ${ }^{1}$ \\ ${ }^{1}$ Dipartimento di Chimica Fisica e Inorganica, University of Bologna, Viale Risorgimento 4, 40136 Bologna, Italy \\ ${ }^{2}$ ISAC - CNR, Via P. Gobetti 101, 40129 Bologna, Italy \\ Correspondence to: M. Carlotti (massimo.carlotti@unibo.it)
}

Received: 18 May 2012 - Published in Atmos. Meas. Tech. Discuss.: 12 September 2012

Revised: 28 January 2013 - Accepted: 28 January 2013 - Published: 20 February 2013

\begin{abstract}
The information load (IL) analysis, first introduced for the two-dimensional approach (Carlotti and Magnani, 2009), is applied to the inversion of MIPAS (Michelson Interferometer for Passive Atmospheric Sounding) observations operated with a 1-dimensional (1-D) retrieval algorithm. The IL distribution of MIPAS spectra is shown to be often asymmetrical with respect to the tangent points of the observations and permits us to define the preferential latitude where the profiles retrieved with a 1-D algorithm should be geo-located. Therefore, defining the geo-location of the retrieved profile by means of the tangent points leads to a "position error". We assess the amplitude of the position error for some of the MIPAS main products and we show that the IL analysis can also be used as a tool for the selection of spectral intervals that, when analyzed, minimize the position error of the retrieved profile. When the temperature $(T)$ profiles are used for the retrieval of volume mixing ratio (VMR) of atmospheric constituents, the $T$-position error (of the order of 1.5 degrees of latitude) induces a VMR error that is directly connected with the horizontal $T$ gradients. Temperature profiles can be externally-provided or determined in a previous step of the retrieval process. In the first case, the IL analysis shows that a meaningful fraction (often exceeding $50 \%$ ) of the VMR error deriving from the 1-D approximation is to be attributed to the mismatch between the position assigned to the external $T$ profile and the positions where $T$ is required by the analyzed observations. In the second case the retrieved $T$ values suffer by an error of $1.5-2 \mathrm{~K}$ due to neglecting the horizontal variability of $T$; however the error induced on VMRs is of minor concern because of the generally small mismatch between the IL distribution of the observations analyzed to retrieve $T$ and those analyzed to retrieve
\end{abstract}

the VMR target. An estimate of the contribution of the $T$ position error to the error budget is provided for MIPAS main products. This study shows that the information load analysis can be successfully exploited in a 1-D context that makes the assumption of horizontal homogeneity of the analyzed portion of atmosphere. The analysis that we propose can be extended to the 1-D inversion of other limb-sounding experiments.

\section{Introduction}

Limb-scanning measurements have proven to be especially suited to study the chemical composition of the atmosphere and to determine the vertical distribution of atmospheric constituents. The limb-scanning technique has been widely used in the past for spectrometers on board aircraft, stratospheric balloons, and orbiting platforms; the latter being quite powerful when they measure the atmospheric emission from polar orbits that allow full geographical and time coverage. A number of this kind of measurements has been realized in the past (e.g. Beer and Glavich, 1989; Waters et al., 1999; Fischer et al., 2008), while others are under consideration by space agencies for the coming years (e.g. ESA, 2008). The retrieval methods adopted for the analysis of limb-scanning observations have evolved in time passing from a "layer by layer" approach (Goldman et al., 1973) to the simultaneous analysis of observations selected from a whole limb-scan sequence (global-fit, Carlotti, 1988). Both strategies assume horizontal homogeneity for the portion of atmosphere spanned by the lines of sight of the observations (1-D assumption). In the case of orbiting platforms the size of this portion of 
atmosphere increases, by effect of the satellite movement, up to a length of about $2000 \mathrm{~km}$ making therefore the horizontal homogeneity assumption a weak point of the analysis. A two-dimensional (2-D) tomographic approach, named geo-fit (Carlotti et al., 2001), was introduced for the MIPAS (Michelson Interferometer for Passive Atmospheric Sounding) experiment in order to overcome the horizontal homogeneity assumption; it can be used for any limb-scanning satellite experiment where the lines of sight of the spectrometer are oriented (and overlap) along the orbit track. With geo-fit the horizontal inhomogeneities are modelled and retrieved through the simultaneous analysis of observations belonging to all the limb-scans measured along a whole orbit. Other 2-D retrieval algorithms (e.g. Livesey and Read, 2000; Steck et al., 2005; Puksīe et al., 2008), exploit 2-D strategies. However, despite of the demonstrated advantages of the 2$\mathrm{D}$ approach, orbiting limb-scanning experiments are mostly analyzed using 1-D algorithms.

In 1-D analyses the geo-location assigned to the retrieved profiles is generally connected with the position of the tangent points of the observations. A common choice is to geolocate the whole profile at the average geographical coordinates of the tangent points of the analyzed scan. The size of the possible geo-location error qualifies the retrieval products but it is not considered to be of concern within the retrieval process because, thanks to the horizontal homogeneity assumption, the profile position is not relevant within the portion of atmosphere spanned by the observations. However, the profile geo-location becomes relevant when it is used to identify coincidence with independent measurements or to calculate average distributions within predefined latitudinal bands (Kiefer et al., 2010).

The inversion of limb-scanning observations usually requires the knowledge of temperature $(T)$ profiles for the retrieval of any atmospheric constituent. The quality of $T$ profiles used in volume mixing ratio (VMR) retrievals is then crucial since their error propagates into the error of the retrieved VMR values. It is therefore necessary to provide an estimate of the errors introduced by 1-D algorithms as a consequence of neglecting the horizontal $T$ gradients. In a VMR retrieval the $T$ profile can be externally-provided or determined in a previous step of the retrieval analysis. In the first case, the external profile is chosen trying to mach at best the time and geo-location of the analyzed observations but an error is expected as a consequence of possible mismatches. In the second case perfect coincidence is achieved (since the profile has been obtained from the same observations) but $T$ profiles retrieved by neglecting horizontal gradients are known to be affected by a systematic error due to the horizontal homogeneity assumption.

In order to analyze the nature and the size of the errors involved in the scenarios described in the previous paragraph, we have studied the 1-D inversion of MIPAS observations. For the purpose we have exploited, within the 1-D context, the information load (IL) analysis (Carlotti and Magnani,
2009) first introduced to study the information contained in consecutive limb-scanning observations when they are analyzed with a 2-D approach (a sample application of IL analysis for 2-D retrievals can be found in Carlotti et al. (2011)). Retrievals on simulated observations have been used to quantify the errors introduced by 1-D algorithms as a consequence of neglecting the horizontal gradients.

This paper is organized as follows: in Sect. 2 we briefly describe the features of the MIPAS experiment that are relevant for this study, and the 1-D retrieval algorithm routinely used in MIPAS ground segment. In Sect. 3 we review the definition and mathematics of the IL and we show that, when exploited within the 1-D context, the IL analysis leads to highlight the definition of "position error". In Sect. 4 we focus on the position error of $T$ profiles and we evaluate the impact of this error on VMR retrievals by means of analyses on simulated observations. In Sect. 5 we discuss the findings of the previous section. Finally, in Sect. 6 we draw conclusions and general considerations about the results of this study.

\section{The MIPAS experiment and operational retrieval methods}

MIPAS is a limb-scanning spectrometer developed by the European Space Agency (ESA) for the study of the atmospheric composition. It was measuring the infra-red atmospheric emission from a nearly polar orbit onboard the ENVISAT satellite. In this paper we refer to the MIPAS nominal mode of observation, operated for the optimized resolution configuration (adopted from January 2005 to April 2012 when ENVISAT ceased operations). This mode consists of consecutive backward-looking limb-scans with the line of sight approximately lying in the orbit plane. Each limb-scan is made of $27 \mathrm{limb}$ views with tangent altitudes ranging from 6 to $70 \mathrm{~km}$ with increasing steps of $1.5,2,3$, and $4 \mathrm{~km}$. The separation between consecutive limb-scans is about $420 \mathrm{~km}$.

MIPAS spectra are analyzed by the ESA ground processor that determines, at the tangent points of each limb-scan, the values of pressure, temperature, and VMR of six key atmospheric species $\left(\mathrm{H}_{2} \mathrm{O}, \mathrm{O}_{3}, \mathrm{HNO}_{3}, \mathrm{CH}_{4}, \mathrm{~N}_{2} \mathrm{O}\right.$ and $\left.\mathrm{NO}_{2}\right)$. Temperature and pressure profiles are first determined and used in the following steps of the retrieval sequence. The MIPAS ground processor uses a 1-D retrieval algorithm (Ridolfi et al., 2000; Raspollini et al., 2006), based on the globalfit approach (Carlotti, 1988). With this strategy a one-to-one correspondence is established between the measured limbscans and the retrieved profiles: since the analyzed portion of atmosphere is assumed to be horizontally homogeneous, all the profiles are conventionally geo-located at the average coordinates of the tangent points of the analyzed limb-scan.

The 2-D geo-fit analysis method was implemented in the operational code GMTR (Carlotti et al., 2006) for routine analysis of MIPAS observations. In this case the 2-D retrieval grid is fully independent from the measurement grid; the 2-D 
grid (typically profiles are retrieved at the average position of each limb-scan) is used to model the horizontal structures of the atmosphere. The MIPAS2D database of level 2 products (Dinelli et al., 2010) generated with the GMTR analysis system has proven to be capable of properly representing the horizontal structures of the atmosphere that are cause of errors when neglected in 1-D algorithms (Kiefer et al., 2010).

Irrespective of the adopted algorithm, the analysis of MIPAS observations is carried out on a selected number of narrow (less than $3 \mathrm{~cm}^{-1}$ wide) spectral intervals, called microwindows (MWs), that carry optimal information on the target quantity (Dudhia et al., 2002). The use of MWs allows to limit the demand on computer resources and to avoid the analysis of spectral regions which are characterized by uncertain spectroscopic data, interference by non-target species, or are influenced by unmodeled effects (Worden et al., 2004).

An exhaustive description of the MIPAS experiment can be found in Fischer et al. (2008).

\section{Information Load analysis for 1-D retrievals}

\subsection{Information Load definitions}

For the analysis of limb-scanning observations with the 2-D approach the atmosphere is partitioned on both the vertical and the horizontal domains (Carlotti et al., 2001). This discretization leads to a web-like picture in which consecutive altitude levels and vertical radii delimit plane atmospheric regions denoted as "cloves". If the simultaneous analysis of several observation geometries is considered, we can assign to each clove the information load scalar quantifier $(\Omega)$ defined as (Carlotti and Magnani, 2009):

$\Omega(q, h)=\left[\sum_{i=1}^{l} \sum_{j=1}^{m} \sum_{k=1}^{n}\left(\frac{\partial S_{i j k}}{\partial q_{h}}\right)^{2}\right]^{1 / 2}$

where:

- $\Omega(q, h)=$ information load of clove $h$ with respect to atmospheric parameter $q$,

- $S_{i j k}=$ spectral signal of observation geometry $i$ at frequency $j$ of the analyzed MW $k$,

$-l=$ number of limb views that cross clove $h$,

- $m=$ number of analyzed MWs in limb view $i$,

- $n=$ number of spectral points in MW $j$.

Equation (1) can be written as:

$\Omega(q, h)=\left[\left(\boldsymbol{k}^{T} \boldsymbol{k}\right)_{h}\right]^{1 / 2}$

where $\boldsymbol{k}$ is the vector containing the derivatives of all the observations that depend on the value of $q$ in clove $h$.
If the observations are affected by different noise levels (e.g. occur in different spectral bands) it is suitable to introduce a new quantifier named Weighted information load $(W \Omega)$ defined as:

$W \Omega(q, h)=\left[\left(\boldsymbol{k}^{T} \mathbf{S}^{-1} \boldsymbol{k}\right)_{h}\right]^{1 / 2}$

where $\mathbf{S}$ is the variance-covariance matrix of the observations relative to all the spectral points that contribute to the information load in clove $h$.

In an unconstrained retrieval analysis the uncertainty on the value of the target quantity $q$ in clove $h$ is given by $1 / W \Omega$ (see Carlotti and Magnani, 2009).

A map of the $W \Omega$ quantifier, calculated for each clove of the 2-D atmospheric discretization (see examples in Sect. 3.2), enables the evaluation of the 2-D distribution of the information load with respect to the geophysical parameter $q$. In these maps:

- the values of $W \Omega$ measure the amount of information contained in the corresponding cloves,

- the spatial distribution of $W \Omega$ indicates the regions of the atmosphere where the information is gathered from when operating a retrieval analysis.

It follows that the IL analysis provides a tool to compare the performance of different observation strategies and/or of different sets of spectral intervals to be analyzed. The $W \Omega$ maps also indicate the optimal strategy to select altitudes and geolocation of the retrieval grid.

In the 2-D context, the observation geometries that cross clove $h$ may come from different limb-scans (see Carlotti and Magnani, 2009) while in the 1-D context they are a subset of the observation geometries of the analyzed limb-scan.

\subsection{The horizontal position error}

Figure 1 shows maps of $W \Omega$ calculated with respect to $\mathrm{O}_{3}$ VMR for a single limb-scan close to the South Pole of MIPAS orbit 30958 (having 96 limb-scans) recorded on 31 January 2008 (we will refer to this orbit in all the examples reported in this paper).

The map in the upper-left panel of Fig. 1 was generated with the set of five MWs used by MIPAS ground segment for the operational retrieval of $\mathrm{O}_{3}$ VMR. The remaining panels of this figure show the $W \Omega$ maps relative to the five individual MWs. The maps of Fig. 1 (as well as all the tests reported in this paper) were calculated using the real observational parameters of a limb-scan close to South Pole; the 2-D atmospheric model was derived combining the profiles retrieved by GMTR from this orbit with climatological data (Remedios et al., 2007) for the minor species. In each map the upper curve represents the Earth's surface. The atmosphere is partitioned with altitude levels separated by $1 \mathrm{~km}$ and radii separated by 1 degree (lines corresponding to levels and radii 


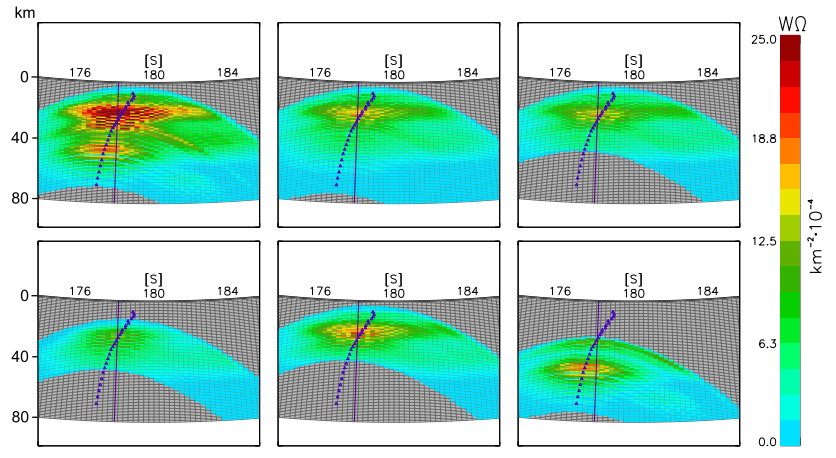

Fig. 1. $W \Omega$ distributions calculated wrt $\mathrm{O}_{3}$ VMR for a limb-scan close to the South Pole of MIPAS orbit 30958. The upper-left panel refers to the set of five MWs used by MIPAS ground segment. The other panels refer to the five individual MWs. In each panel the upper curve represents the Earth's surface. The atmosphere (expanded by a factor 10 with respect to the Earth's radius) extends downwards up to $80 \mathrm{~km}$ (the altitudes are reported in the left axes). Blue triangles mark the tangent points of the observations. The blue solid lines show the geo-location of the profile retrieved by the 1-D analysis. Orbital coordinates are reported in degrees. The position of South Pole is marked by [S]. The satellite position is on the right hand side of the image and moves counterclockwise.

can be seen in the portions of maps that are not covered by observations). Blue triangles mark the tangent points of the observations while the blue solid line shows the average horizontal position of the tangent points that is the position where the profile retrieved by the 1-D analysis of this limb-scan is geo-located. Values of the orbital coordinate (OC) (defined as the polar angle originating at the North Pole and spanning the orbit plane over its 360 degrees extension) are also reported in Fig. 1.

The intensity and the 2-D distribution of $W \Omega$ highlight the locations where information will be gathered from when retrieving a profile using the considered observations; hence they indicate the "optimal" geo-location for the retrieved profiles, being the one that is where the bulk of the atmospheric information has derived. The upper-left panel of Fig. 1 shows that, in the horizontal domain, the $W \Omega$ distribution relative to the full set of $\mathrm{O}_{3} \mathrm{MWs}$ is broad and asymmetric with respect to both the tangent points and the position where the 1-D retrieved profile is geo-located. Therefore, an effective error in the geo-location of the VMR profile is expected. The $W \Omega$ distribution of the full set of MWs can be exploited to define, at each altitude, a more correct geo-location of the retrieved profile. A natural criterion could be to use the position of the $W \Omega$ maximum. However (as it can be appreciated in Fig. 1), the $W \Omega$ distributions do not show unambiguous maxima in their horizontal extension (this is a consequence of the finite atmospheric discretization). A reasonable assumption is to define the geo-location of the profile at a given altitude as the position of the median of the $W \Omega$ distribution at that altitude. The median criterion has the advantage of taking into account both the intensity and the horizontal spread of $W \Omega$. However, the median criterion (as any other criterion) tends to loose significance at altitudes where the $W \Omega$ intensity is weak. The altitude limit depends on both the target and the atmospheric scenario (it is within 40 and $50 \mathrm{~km}$ for MIPAS targets).

The mismatch (in latitudinal degrees) between the position derived from the layout of the information used for the retrieval and the position where the profile is assigned by the 1-D analysis defines what we call the "position error". In order to clarify this concept we refer to Fig. 2a. Here, red crosses mark (at each altitude of the vertical discretization) the position of the medians of the $W \Omega$ distribution reported in the upper-left panel of Fig. 1. The red line identifies the positions where (following the median criterion) we assign the retrieved $\mathrm{O}_{3}$ profile. This line is generated by a polynomial that fits the red crosses. Blue triangles show the tangent points of this limb-scan while the vertical line marks the position where the retrieved profile is assigned by the 1-D analysis. The position error is given by the distance between the red line and the vertical line. The concept of "position error" was highlighted previously by von Clarmann et al. (2009) that studied the 2-D averaging kernel (AK) of 1-D retrievals (see Sect. 5.3).

The $W \Omega$ distributions depend on the physical and chemical properties of the atmosphere. Therefore size and shape of the position errors change for each limb-scan along the orbit. The envelop of all the position errors along the orbit is given by the green lines in Fig. 2b. In the same panel the red line shows the profile of the average position error. An overall assessment of the average position error (along the orbit) for five MIPAS main targets (namely $\mathrm{H}_{2} \mathrm{O}, \mathrm{O}_{3}, \mathrm{HNO}_{3}, \mathrm{CH}_{4}$, and $\mathrm{N}_{2} \mathrm{O}$ ) is given in Fig. $2 \mathrm{c}$.

In the representation of Fig. 1, the satellite moves counterclockwise so that the satellite position is on the right hand side of the image. Since MIPAS looks backward, the $W \Omega$ distributions are generally offset toward higher values of the OC with respect to the tangent points because the opacity of the atmosphere tends to increase while moving away from the satellite along the line of sight. This effect is confirmed by predominant positive position errors in the plots of Fig. 2 .

In a first approximation the position error of the retrieved VMR profiles can be neglected because, due to the horizontal homogeneity assumption, the retrieved profile is valid anywhere within the analyzed portion of the atmosphere. However this type of error may acquire significance when the geo-location of the retrieved profiles is used to identify coincidences (e.g. with independent measurements or with particular atmospheric events) and when the position is used to average profiles within predefined latitudinal bands (see e.g. Kiefer et al., 2010).

Finally, in Fig. 1 we notice that the maps relative to the individual MWs permit to identify the spread of $W \Omega$ and the asymmetry elements that are introduced by each MW in the overall map of the upper-left panel. The evaluation of these 

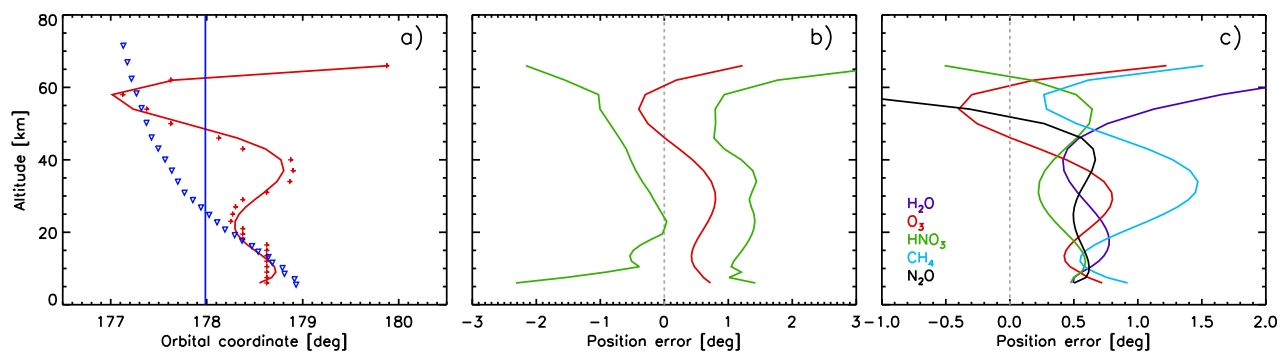

Fig. 2. (a): red crosses mark the position of the median of the $W \Omega$ distributions reported in upper-left panel of Fig. 1 . A polynomial fit to the crosses (red line) identifies the positions where we assign the retrieved $\mathrm{O}_{3}$ profile. Blue triangles mark the tangent points of the observations. The blue vertical line is the geo-location of the profile adopted by the 1-D analysis. (b): green lines delimit the envelop of the $\mathrm{O}_{3}$ position errors along the orbit, the red line is the average position error of $\mathrm{O}_{3}$ profiles. (c): average position error (along the orbit) for five MIPAS main targets.

properties suggests that sharpness and symmetry of their $W \Omega$ distribution should be a criterion for the selection of MWs that, when analyzed, minimize the position error of the retrieved profile.

\section{The temperature-position error and its propagation}

\subsection{The temperature position error}

Figure 3 shows the $W \Omega$ distribution with respect to $T$ for the same limb-scan of Fig. 1, calculated with two different sets of MWs. The left panel refers to the set of MWs used by MIPAS ground segment for the retrieval of $T$ profiles, the right panel refers to the MWs used for the retrieval of $\mathrm{O}_{3}$ VMR (see below in this section). In Fig. 4, the left panel shows the same quantities as the left panel of Fig. 2 but for the retrieval of $T$ from this limb-scan. The right panel of Fig. 4 reports the profile of the average $T$-position errors and their envelop calculated over the entire orbit (using the format as in Fig. 2b). Figures 3 (left panel) and 4 show that, in the case of $T$, the asymmetry of the $W \Omega$ distribution is quite strong and causes a significant position error (of the order of 1-1.5 degrees) in a wide range of altitudes. Temperature profiles are therefore among the MIPAS products most affected by this kind of error.

A further issue arises if we consider that temperature is not horizontally homogeneous within the sampled portion of atmosphere. Actually, if a $T$ profile is used as an input in subsequent analyses of VMR targets, the presence of horizontal gradients makes the $T$ profile at the position where it is assigned different from the one at the position where $T$ is required by the forward model to simulate the analyzed observations. The latter position can be derived from the $W \Omega$ distributions with respect to $T$ for the MWs that are analyzed in the VMR retrieval. The right panel of Fig. 3 shows this distribution calculated (for the same limb-scan as in the left panel) for the MWs analyzed in the retrieval of $\mathrm{O}_{3}$ VMR. The median criterion, applied to the latter distribution, defines the
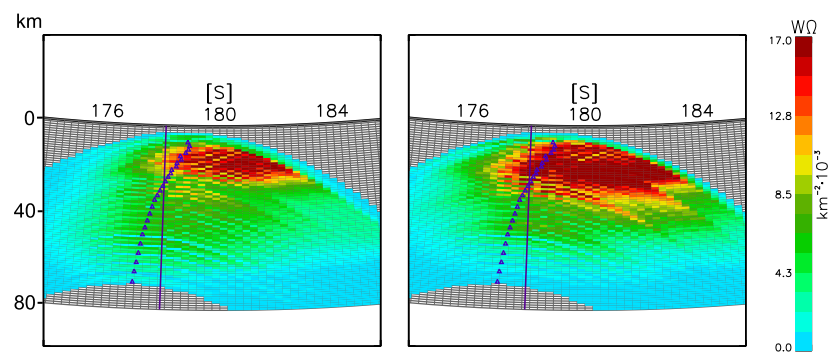

Fig. 3. $W \Omega$ distributions with respect to $T$ calculated with the set of MWs used by the MIPAS ground segment for the retrieval of $T$ profiles (left panel), and for the retrieval of $\mathrm{O}_{3}$ VMR profiles (right panel).

positions where $T$ is used by the forward model (see Sect. 4.3 and Fig. 7).

The above described situation requires us to evaluate the propagation of the $T$-position error into the VMR retrievals and to assess its importance with respect to the total error expected as a consequence of not modelling the horizontal variability in 1-D retrievals. We notice here that no propagation of the $T$-position error is expected if $T$ and the VMR target are jointly retrieved (as in Carlotti et al., 2006).

\subsection{Simulated retrievals strategy}

The retrieval analysis operated on simulated observations (simulated retrieval) is used in this work to assess the errors that are introduced when neglecting the horizontal atmospheric variability. The steps of our simulated retrievals are:

1. A 2-D forward model generates synthetic spectra for the whole orbit using a set of reference profiles that describe the horizontal structures of the atmosphere. For the purpose we have used profiles retrieved from a previous analysis of the considered orbit; the layout of the reference profiles is determined by the $\mathrm{OC}$ of the tangent points of each limb-scan. Simulated observations 


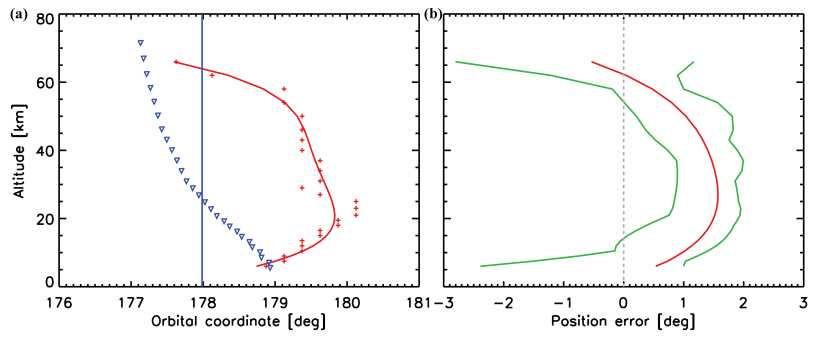

Fig. 4. Left panel: as (a) of Fig. 2 but for $T$. Right panel: as (b) of Fig. 2 but for $T$.

are obtained by adding random noise to the synthetic spectra. In order to minimize the contribution of random errors (see step 3) the amplitude of spectral noise is reduced by a factor 40 with respect to the values of the real MIPAS observations.

2. For each limb-scan the 1-D retrieval of the target quantity is carried out on its simulated observations. In this step the fields of both $T$ and VMRs are kept horizontally homogeneous along the iterations of the retrieval (in accordance with the 1-D assumption). All the geometrical and auxiliary data are the same as at point 1 . The initial guess of the target quantity is obtained by applying random perturbations to the reference profile. No a-priori information is used in the retrieval.

3. The difference between retrieved and reference profiles provides the error that is mainly to be attributed to the horizontal homogeneity assumptions made on both $T$ and VMR atmospheric fields (since the spectral noise is negligible and all other assumptions are maintained as in the 2-D simulation of the observations).

In order to assess the propagation of the $T$-position error into VMR retrievals, we distinguish the case in which $T$ profiles are of external origin (e.g. taken from climatology) from the case of $T$ profiles determined in a previous step of the retrieval analysis.

\subsection{Propagation to VMR retrievals adopting externally-provided $T$ profiles}

The latitudinal gradient of a geophysical parameter is sounded by MIPAS with opposite sign in the two halves of the orbit (descending half with OC running from 0 to 180 degrees, and ascending half from 180 to 360 degrees). On the other hand (as a rough approximation) minor variability is expected in the atmosphere when moving along the longitudes of a given latitudibal band. For these reasons the error due to neglecting the horizontal variability in 1-D retrievals is expected to show opposite sign in the two halves of the orbit. The map in the upper panel of Fig. 5 shows the horizontal $T$ gradients as derived from the GMTR analysis of MIPAS
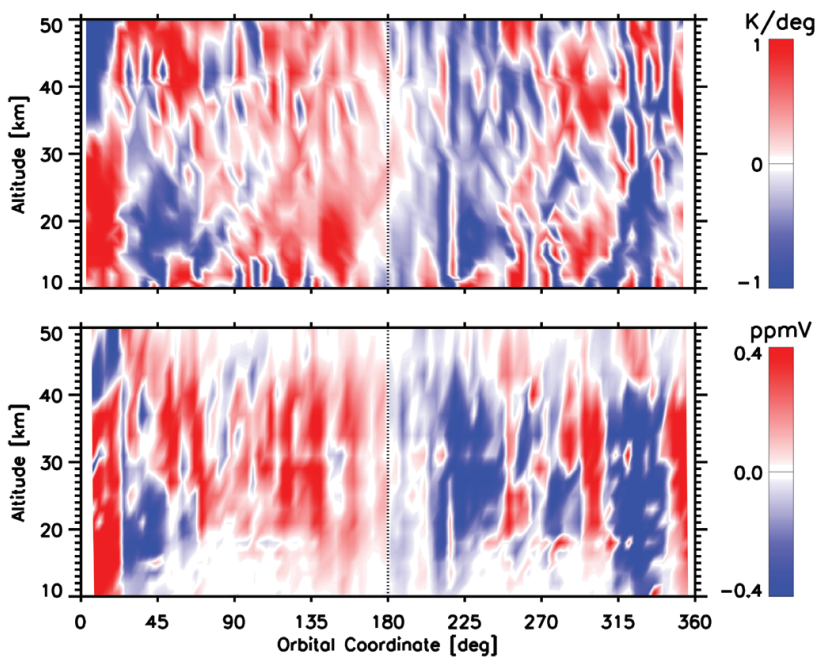

Fig. 5. Upper panel: horizontal $T$ gradients derived from the GMTR analysis of MIPAS orbit 30958 as seen by MIPAS lines of sight, plotted as a function of altitude and OC. Lower panel: difference between the retrieved and the reference values for a simulated retrieval of $\mathrm{O}_{3}$ VMR that uses horizontally homogeneous $T$ fields derived from the $T$ profiles of orbit 30958 (see text).

orbit 30958. In this analysis the 2-D retrieval grid was defined by the tangent points of the observations. Gradients of Fig. 5 are calculated in the direction opposite to the satellite movement and are reported as a function of altitude and OC. In this map the opposite sign of the horizontal $T$ gradients, along the MIPAS lines of sight, can be appreciated at latitudes symmetrically displaced with respect to the $180 \mathrm{de}-$ grees (South Pole) axis (compare e.g. southern mid-latitude regions around OC 135 and OC 225).

The $T$ profiles that generate the map in the upper panel of Fig. 5 (reference profiles) have been used to generate simulated observations. In this specific test the reference profiles are also used as externally-provided $T$ profiles in the VMR retrieval. Target VMRs are then retrieved using a $T$ field that is made horizontally homogeneous by replicating the reference profile over the full portion of the sampled atmosphere. The reference profiles, as such, are not affected by uncertainty but (as stated in point 1 of Sect. 4.2) their position is identified by the tangent points of the analyzed limb-scan. The lower panel of Fig. 5 shows the difference between the retrieved and the reference $\mathrm{O}_{3}$ VMR values obtained with the 1-D retrieval on these simulated observations. The comparison between the two panels of Fig. 5 highlights the correlation, in both sign and amplitude, between $T$ gradients and $\mathrm{O}_{3}$ VMR errors. The altitude axes of Fig. 5 are restricted to an interval where the correlations can be better appreciated (outside altitudes have ozone VMRs so small that the values of absolute errors is dominated by other effects). An overall assessment of these errors can be found in the left panel of Fig. 6 where the blue line shows the RMS of the difference 


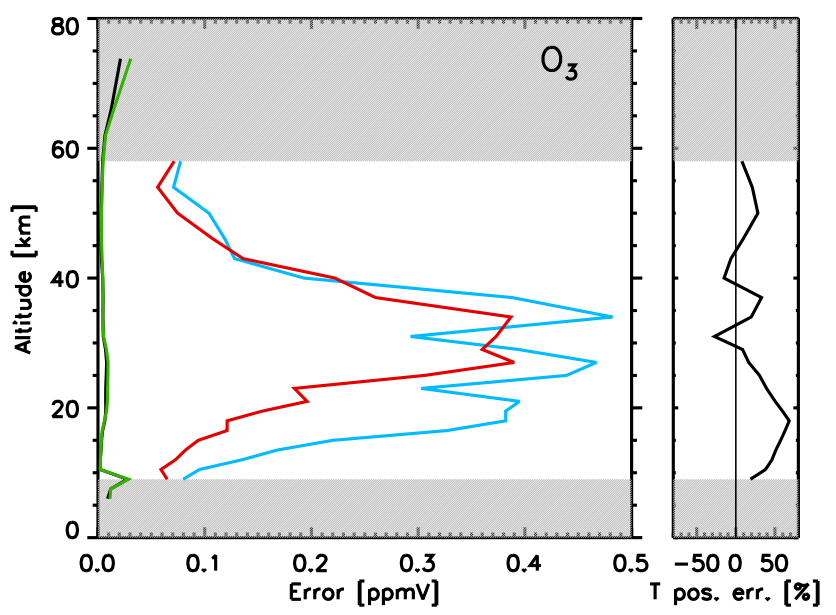

Fig. 6. Left panel: blue line is the RMS of the difference (retrievedreference) $\mathrm{O}_{3} \mathrm{VMR}$ profiles that generate the map in the lower panel of Fig. 5, red line is the RMS of the difference profiles when the $T$ position error has been corrected with the interpolation strategy between the externally-provided $T$ profiles, black and green lines are control tests used to delimit the altitude range of blue and red lines (see text). Right panel: percent contribution of the $T$-position error (blue-minus red-curve in the left panel) with respect to the entire error due to the horizontal homogeneity assumption (blue curve in the left panel). Altitude regions where the lack of stability is intrinsic in the retrieval are shaded (see Sect. 4.3).

between retrieved and reference $\mathrm{O}_{3}$ VMR profiles, calculated over all the limb-scans of the considered orbit (the red line of this panel is introduced below in this section).

In the left panel of Fig. 6 the black line shows the RMS obtained when the $\mathrm{O}_{3}$ VMR is derived using the 2-D retrieval (that is when horizontal gradients are modeled) whereas the green line refers to the 1-D retrieval operated on observations simulated with horizontally homogeneous atmosphere. The black and green lines are just control tests as they are expected not to deviate from zero; their divergence from the $y$ axis indicates an intrinsic lack of stability of the inversion process at altitudes where $W \Omega$ gets next to zero. Therefore in Fig. 6 (as well as in Figs. 8 and 9 below) we plot the results of our tests only in the altitude range where the control tests are stable. The altitude regions where the lack of stability is intrinsic in the retrieval are shadowed in these figures.

In order to discuss the propagation of the $T$-position error in presence of $T$ horizontal gradients we consider again the limb-scan near the South Pole. In the left panel of Fig. 7 the blue plots show the $T$ profile position (at tangent points) of the examined (central) and of the adjacent scans, while the red line shows the position where the $T$ profile is required by the forward model to simulate the $\mathrm{O}_{3}$ observations of the central scan, that is the median of the $W \Omega$ distribution with respect to $T$ calculated using the $\mathrm{O}_{3} \mathrm{MWs}$ (see the right panel of Fig. 3). Considering the presence of horizontal $T$ gradients, temperatures at the position of the red line are different
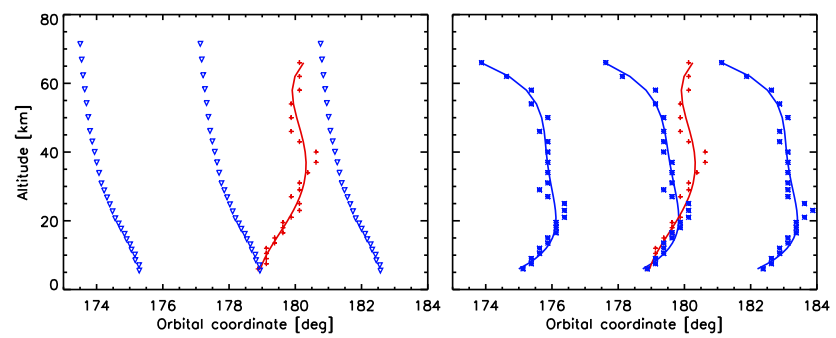

Fig. 7. Left panel: the red plots identify (with the same format as in Fig. 2) the positions where $T$ is required by the forward model to simulate the $\mathrm{O}_{3} \mathrm{MWs}$, blue triangles identify the position of the externally-provided $T$ profiles used to interpolate $T$ on the red line. Right panel: red plots are as in the left panel, blue plots identify the positions of the retrieved $T$ profiles used to interpolate $T$ on the red line.

from the ones at the position of the central blue line. Therefore a $T$ profile suitable for simulating the observations of this limb-scan can be calculated by interpolating between the surrounding (externally-provided) reference $T$ profiles on the position of the red line.

We have repeated the $\mathrm{O}_{3}$ VMR retrieval of the full orbit by replacing the reference $T$ profiles with the interpolated ones. The red curve in the left panel of Fig. 6 shows the RMS of the difference between retrieved and reference profiles obtained in this case. The right panel of Fig. 6 shows the percent contribution of the $T$-position error (blue minus red curves in the left panel of the same figure) with respect to the entire error due to the horizontal homogeneity assumption (blue curve in the left panel).

The same analysis has been carried out for other MIPAS main targets (see Sect. 2); results are shown in Fig. 8 with the same format as for $\mathrm{O}_{3}$ in Fig. 6. It can be seen in Figs. 6 and 8 that, at altitudes below $30 \mathrm{~km}$, the $T$-position error is responsible for a meaningful fraction (often exceeding $50 \%$ ) of the error due to neglecting the horizontal gradients.

\subsection{Propagation to VMR retrievals adopting retrieved $T$ profiles}

We consider now the case in which VMR retrievals are carried out using $T$ profiles that have been determined in a previous step of the analysis (as in MIPAS ground segment). As a first step we have used the simulated retrieval strategy to evaluate the error propagated on $T$ profiles by the horizontal homogeneity assumption. The upper-left panel of Fig. 9 shows the RMS of the difference between retrieved and reference $T$ profiles calculated over all the limb-scans of the orbit. As discussed in Sect. 4.1, the geo-location of the retrieved $T$ profiles is identified by the median of the $W \Omega$ distribution with respect to $T$ of the analyzed MWs (see the left panel of Fig. 4 for the limb-scan close to the South Pole).

In this case, in order to correct for the $T$-position error, an approximation of the $T$ profile required by the forward 

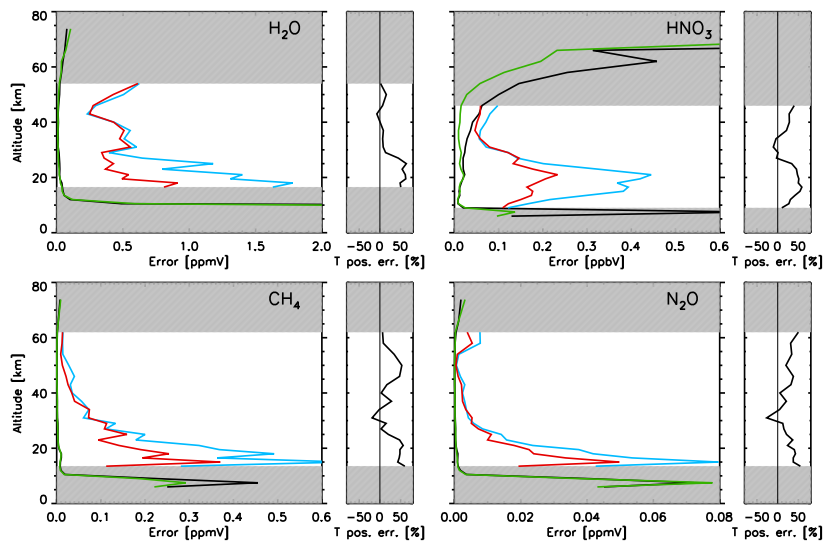

Fig. 8. Same quantities as in Fig. 6 for $\mathrm{H}_{2} \mathrm{O}, \mathrm{HNO}_{3}, \mathrm{CH}_{4}$, and $\mathrm{N}_{2} \mathrm{O}$ (see labels).

model to simulate the limb-scan close to the South Pole can be calculated by interpolating the retrieved $T$ profiles on the positions of the red line of Fig. 7. The right panel of this figure also reports, in blue, the position of the adjacent $T$ profiles determined as described in Sect. 4.1 (see Fig. 4).

By analogy with Figs. 6 and 8, the other five panels of Fig. 9 refer to the VMR targets (see labels) and report the RMS of the difference between retrieved and reference profiles when the retrieved $T$ profiles are used as such (blue lines) and when the $T$-position errors have been corrected with the interpolation strategy between retrieved $T$ profiles (red lines).

\section{Discussion}

\subsection{Propagation of $\boldsymbol{T}$-position error}

In Fig. 9 we notice that: (i) the horizontal homogeneity assumption leads to significant errors in the retrieved $T$ profiles (of the order of 1-2 K), (ii) the propagation of these $T$ errors in the retrieved VMR profiles is surprisingly small: the amplitude of blue lines in Fig. 9 is of the order of $50 \%$ of the corresponding lines in Figs. 6 and 8, (iii) the interpolation strategy implemented to correct for the $T$-position error does not lead (with the exception of $\mathrm{O}_{3}$ ) to appreciable improvements in the retrieved VMR profiles.

In order to interpret these results we recall that the 1-D retrieval system looks for the profile that better fits the analyzed observations. In the presence of horizontal structures, the reference profiles (that is the profiles used to generate the simulated observations; see Sect. 4.2) do not produce the best fit of the observations. Actually, if we run the simulated $T$ retrieval (upper-left panel of Fig. 9) using the unperturbed reference profiles as initial guess, the value of the $\chi^{2}$ calculated with the initial guess is, on average, 34 times larger than the value calculated at convergence. We then deduce that the 1-D retrieval leads to approximate an "effective" profile that
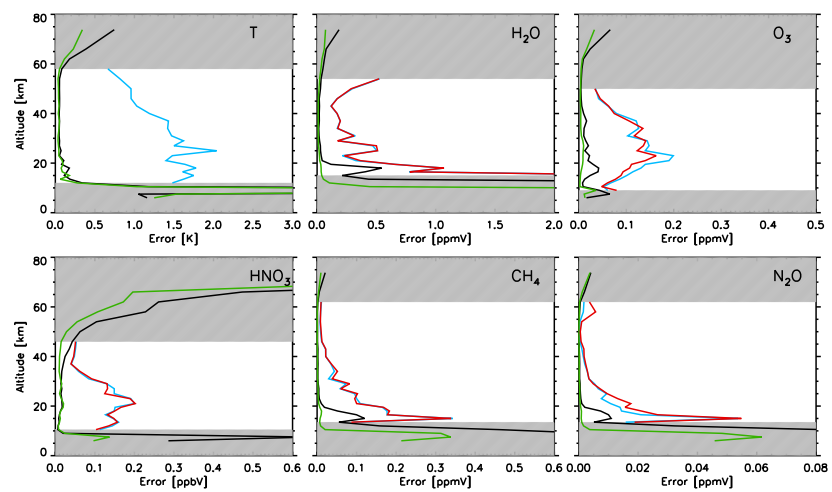

Fig. 9. Upper-left panel: blue line is the RMS of the difference between retrieved and reference $T$ profiles calculated over all the orbit, black and green lines have the same meaning as in Figs. 6 and 8. Other panels: same quantities as in Fig. 6 for $\mathrm{H}_{2} \mathrm{O}, \mathrm{O}_{3}, \mathrm{HNO}_{3}$, $\mathrm{CH}_{4}, \mathrm{~N}_{2} \mathrm{O}$ VMRs obtained using the retrieved $T$ profiles; the red line is the RMS of the difference profiles when the $T$-position error has been corrected with the interpolation strategy between retrieved $T$ profiles.

simulates at best the effect induced by horizontal variability onto the observed spectra. In the case of $T$ we can say that the behavior of an atmosphere characterized by horizontal $T$ variability is simulated by a horizontally homogeneous atmosphere described by its "effective" $T$ profile. The retrieved $T$ profile is then more suitable than the reference profile to simulate the radiative properties of the atmosphere within a VMR retrieval. These considerations explain the smaller amplitude of blue lines in Fig. 9 with respect to the corresponding lines in Figs. 6 and 8.

As for the correction of the $T$-position error, Fig. 7 is representative of the average conditions along the full orbit. In this figure we notice that in the case of the externallyprovided $T$ profiles (left panel) the median of the $W \Omega$ distribution with respect to $T$ of the analyzed MWs is well displaced from the position where the $T$ profile is geo-located on the basis of the tangent points of the observations. Therefore the interpolated $T$ profile is expected to differ significantly from the external profile by effect of the horizontal $T$ gradients. This explains the improvements observed in Figs. 6 and 8 when using the interpolated profiles. In the case of retrieved $T$ profiles (right panel of Fig. 7) the median of the $W \Omega$ distribution with respect to $T$ of the $\mathrm{O}_{3}$ MWs is close to the position that should be attributed to the retrieved $T$ profile, at least up to the altitudes where the median of the $W \Omega$ distribution starts loosing its physical significance (see Sect. 3.2). The changes introduced by the interpolation process are therefore of minor entity. This explains the small differences observed, in Fig. 9, when using the interpolated profiles. 


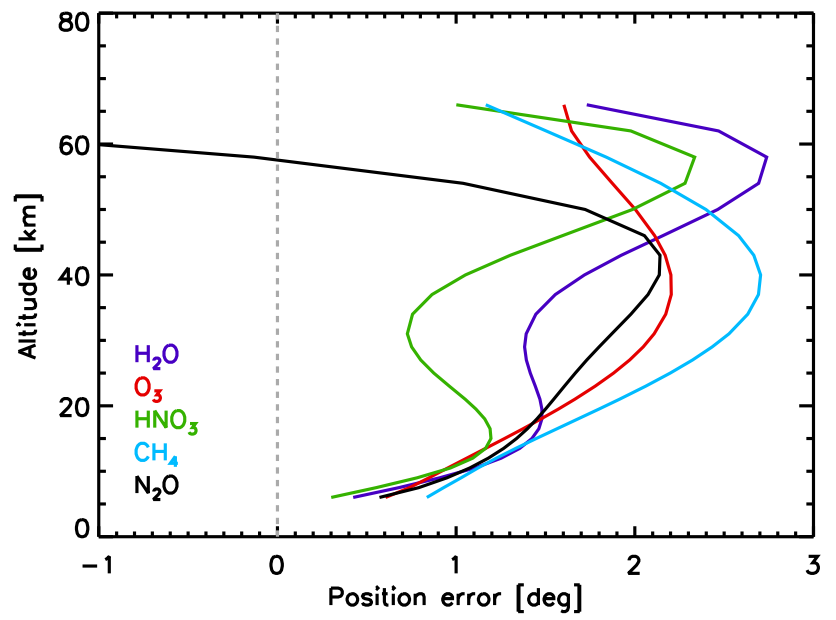

Fig. 10. Average position error of the $W \Omega$ distribution with respect to $T$ for the sets of MWs that are analyzed for the retrieval of $\mathrm{H}_{2} \mathrm{O}$, $\mathrm{O}_{3}, \mathrm{HNO}_{3}, \mathrm{CH}_{4}$, and $\mathrm{N}_{2} \mathrm{O}$ VMRs.

\subsection{Components of the temperature-position error}

The maps in Fig. 3 are relative to two different sets of MWs and report the corresponding distribution of $W \Omega$ with respect to $T$. In both cases it can be seen that the asymmetry with respect to the tangent points is quite large. This peculiarity is common to all the sets of analyzed MWs regardless of the target quantity and, therefore, of the MIPAS band where the observations occur. The common asymmetry is also highlighted in Fig. 10 that shows the average position error of the $W \Omega$ distribution with respect to $T$ for the sets of MWs that are analyzed by the ground segment for the retrieval of the five MIPAS targets considered in this paper.

We have investigated the origin of the strong asymmetry of the $W \Omega$ distributions with respect to $T$ starting from the consideration that, in the radiative transfer process, the values of $T$ mainly affect the behavior of the source function (the Planck function in our case of local thermodynamic equilibrium assumption).

In order to understand the relative weight of the source function component in the derivatives of Eq. (1), in Fig. 11 we show the $W \Omega$ distribution calculated for the same observations of the left panel of Fig. 3 but forcing to zero the derivatives of the Planck function component. Note that, to better display the $W \Omega$ distribution, in Fig. 11 the color scale is expanded by a factor 4 with respect to Fig. 3. The intensities and the symmetry observed in Fig. 11 as compared to those in the left panel of Fig. 3, indicate that the derivatives of the Planck function are the main factor responsible for the observed asymmetry of all the $W \Omega$ distributions with respect to $T$. They overwhelm the $T$ derivatives of other elements of the radiative transfer (e.g. the cross-section of the analyzed transitions).

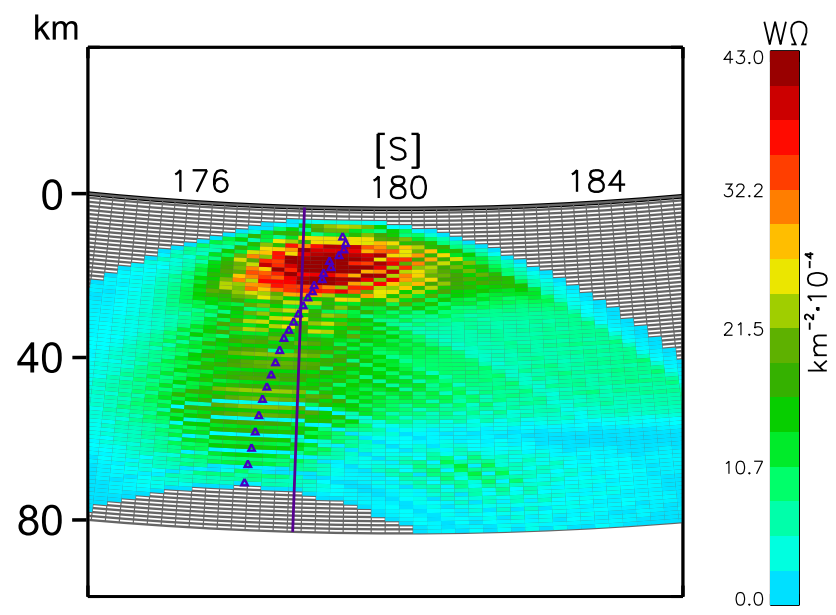

Fig. 11. $W \Omega$ distribution calculated for the same observations of the left panel of Fig. 3 but forcing to zero the derivatives of the Planck function.

\subsection{Position error and averaging kernel}

Further evidence of the position errors is provided by the 2-D averaging kernel (AK) relative to a 1-D retrieval (von Clarmann et al., 2009). In this case the position error is highlighted by the offset between the position assigned (apriori) to the retrieved profile and the position identified (aposteriori) by the horizontal distribution of the 2-D AK. The size of the position errors estimated using the 2-D AK is consistent with the one estimated using the IL analysis. However, the $\mathrm{AK}$ is a property of retrieved profiles while the information load is a property of the observations. This makes the IL analysis suitable to identify in advance the layout of the profile that will produce a 2-D AK which is symmetrical with respect to its position. Furthermore, the 2-D AK cannot provide a criterion for the selection of MWs that, when analyzed, minimize the position error of the retrieved profile (see Sect. 3.2) because the AK calculation requires the inversion of a matrix that, when defined for a single MW, is often singular.

\section{Conclusions}

We have used the information load analysis to study the 1D inversion of MIPAS observations. The information load associated with the observations of a single limb-scan is often characterized, in the horizontal domain, by asymmetries with respect to the position of the tangent points. As a consequence, a mismatch exists between the position where the retrieved profiles are assigned by 1-D analyses and the position where the information has been gathered from to derive them. The mismatch, that we call position error, occurs for all the MIPAS targets but is especially pronounced in the case of $T$ for which it reaches 1.5-2 latitude degrees. In the 
presence of horizontal $T$ gradients, the $T$-position error is expected to propagate into the retrieved VMR profiles. Simulated retrievals have been used to demonstrate the propagation of the $T$-position error and to assess its magnitude. In the case of externally-provided $T$ profiles the propagated error is significant especially below $40 \mathrm{~km}$ where it can exceed $50 \%$ of the error due to neglecting the horizontal structures. If $T$ profiles are derived in a first step of the retrieval analysis (this is the case of MIPAS routine retrievals) they suffer by an error (of the order of 1.5 degrees) due to the horizontal homogeneity assumption that affects the accuracy of these profiles if they have to be considered as retrieval products. However, the 1-D retrieval leads to determine "effective" $T$ profiles that simulate the effect induced by horizontal variability onto the observed spectra. For this reason the retrieved $T$ profiles provide satisfactory performance within the VMR retrievals. Furthermore, if $T$ profiles are first retrieved the propagation of the $T$-position error into the target VMR profiles is of minor entity. This unexpected result is explained by the IL analysis that shows good matching between the position of the retrieved $T$ profile and the position where this profile is required by the VMR analysis.

We have shown that the information load analysis also provides a tool for the selection of observations (MWs in the case of MIPAS) that, when analyzed, minimize the position error of the retrieved profile.

A strong asymmetry is common to all the horizontal distributions of $W \Omega$ with respect to $T$, independent from the analyzed observations. We have shown that the $T$ dependence of the Planck function is responsible for this asymmetry rather than the $T$ dependence of other elements of the radiative transfer (e.g. the cross-section of the analyzed transitions).

The analysis strategy that we have used in this study can be extended to the 1-D inversion of observations acquired by any limb sounder.

Acknowledgements. E. A. acknowledges the support by ESA through the CHIMTEA project within the framework of the Changing Earth Science Network Initiative.

Edited by: J. Joiner

\section{References}

Beer, R. and Glavich, T. A.: Remote sensing of the troposphere by infrared emission spectroscopy, in: Advanced Optical Instrumentation for Remote Sensing of the Earth's Surface from Space, edited by: Duchossois, G., Herr, F. L., and Zander, R., Proc. SPIE, 1129, 42-51, 1989.

Carlotti, M.: Global-fit approach to the analysis of limb-scanning atmospheric measurements, Appl. Optics, 27, 3250-3254, 1988.

Carlotti, M. and Magnani, L.: Two-dimensional sensitivity analysis of MIPAS observations, Opt. Express, 17, 5340-5357, 2009.
Carlotti, M., Dinelli, B. M., Raspollini, P., and Ridolfi, M.: Geofit approach to the analysis of limb-scanning satellite measurements, Appl. Optics, 40, 1872-1885, 2001.

Carlotti, M., Brizzi, G., Papandrea, E., Prevedelli, M., Ridolfi, M., Dinelli, B. M., and Magnani, L.: GMTR: Two-dimensional geofit multitarget retrieval model for Michelson Interferometer for Passive Atmospheric Sounding/Environmental Satellite observations, Appl. Optics, 45, 716-727, 2006.

Carlotti, M., Castelli, E., and Papandrea, E.: Two-dimensional performance of MIPAS observation modes in the uppertroposphere/lower-stratosphere, Atmos. Meas. Tech., 4, 355365, doi:10.5194/amt-4-355-2011, 2011.

Dinelli, B. M., Arnone, E., Brizzi, G., Carlotti, M., Castelli, E., Magnani, L., Papandrea, E., Prevedelli, M., and Ridolfi, M.: The MIPAS2D database of MIPAS/ENVISAT measurements retrieved with a multi-target 2-dimensional tomographic approach, Atmos. Meas. Tech., 3, 355-374, doi:10.5194/amt-3-355-2010, 2010.

Dudhia, A., Jay, V. L., and Rodgers, C. D.: Microwindow selection for high-spectral-resolution sounders, Appl. Optics, 41, 36653673, 2002.

Fischer, H., Birk, M., Blom, C., Carli, B., Carlotti, M., von Clarmann, T., Delbouille, L., Dudhia, A., Ehhalt, D., Endemann, M., Flaud, J. M., Gessner, R., Kleinert, A., Koopman, R., Langen, J., López-Puertas, M., Mosner, P., Nett, H., Oelhaf, H., Perron, G., Remedios, J., Ridolfi, M., Stiller, G., and Zander, R.: MIPAS: an instrument for atmospheric and climate research, Atmos. Chem. Phys., 8, 2151-2188, doi:10.5194/acp-8-2151-2008, 2008.

Goldman, A., Murcray, D. G., Murcray, F. J., Williams, W. J., and Brooks, J. N.: Distribution of water vapor in the stratosphere as determined from balloon measurements of atmospheric emission spectra in the 24-29- $\mu$ m region, Appl. Optics, 12, 1045-1053, 1973.

ESA: Candidate Earth Explorer Core Missions - Report for Assessment: PREMIER - PRocess Exploitation through Measurements of Infrared and millimetre-wave Emitted Radiation, SP-1313/5, ESA Publications Division, ESTEC, Keplerlaan 1, 2200 AG Noordwijk, The Netherlands, 2008.

Kiefer, M., Arnone, E., Dudhia, A., Carlotti, M., Castelli, E., von Clarmann, T., Dinelli, B. M., Kleinert, A., Linden, A., Milz, M., Papandrea, E., and Stiller, G.: Impact of temperature field inhomogeneities on the retrieval of atmospheric species from MIPAS IR limb emission spectra, Atmos. Meas. Tech., 3, 1487-1507, doi:10.5194/amt-3-1487-2010, 2010.

Livesey, N. J. and Read, W. G.: Direct Retrieval of Line-of-Sight Atmospheric Structure from Limb Sounding Observations, Geophys. Res. Letters, 27, 891-894, 2000.

Puķīte, J., Kühl, S., Deutschmann, T., Platt, U., and Wagner, T.: Accounting for the effect of horizontal gradients in limb measurements of scattered sunlight, Atmos. Chem. Phys., 8, 3045-3060, doi:10.5194/acp-8-3045-2008, 2008.

Raspollini, P., Belotti, C., Burgess, A., Carli, B., Carlotti, M., Ceccherini, S., Dinelli, B. M., Dudhia, A., Flaud, J.-M., Funke, B., Höpfner, M., López-Puertas, M., Payne, V., Piccolo, C., Remedios, J. J., Ridolfi, M., and Spang, R.: MIPAS level 2 operational analysis, Atmos. Chem. Phys., 6, 5605-5630, doi:10.5194/acp6-5605-2006, 2006.

Remedios, J. J., Leigh, R. J., Waterfall, A. M., Moore, D. P., Sembhi, H., Parkes, I., Greenhough, J., Chipperfield, M. P., and 
Hauglustaine, D.: MIPAS reference atmospheres and comparisons to V4.61/V4.62 MIPAS level 2 geophysical data sets, Atmos. Chem. Phys. Discuss., 7, 9973-10017, doi:10.5194/acpd-79973-2007, 2007.

Ridolfi, M., Carli, B., Carlotti, M., von Clarmann, T., Dinelli, B. M., Dudhia, A., Flaud, J.-M., Höpfner, M., Morris, P. E., Raspollini, P., Stiller, G., and Wells, R. J.: Optimized forward model and retrieval scheme for MIPAS near-real-time data processing, Appl. Optics, 39, 1323-1340, 2000.

Steck, T., Höpfner, M., von Clarmann, T., and Grabowski, U.: Tomographic retrieval of atmospheric parameters from infrared limb emission observations, Appl. Optics, 44, 3291-3301, 2005. von Clarmann, T., De Clercq, C., Ridolfi, M., Höpfner, M., and Lambert, J.-C.: The horizontal resolution of MIPAS, Atmos. Meas. Tech., 2, 47-54, doi:10.5194/amt-2-47-2009, 2009.
Waters, J., Read, W. G., Froidevaux, L., Jarnot, R. F., Cofield, R. E., Flower, D. A., Lau, G. K., Pickett, H. M., Santee, M. L., Wu, D. L., Boyles, M. A., Burke, J. R., Lay, R. R., Loo, M. S., Livesey, N. J., Lungu, T. A., Manney, G. L., Nakamura, L. L., Perun, V. S., Ridenoure, B. P., Shippony, Z., Siegel, P. H., Thurstans, R. P., Harwood, R. S., Pumphrey, H. C., and Filipiak, M. J.: The UARS and EOS Microwave Limb Sounder Experiments, J. Atmos. Sci., 56, 194-218, 1999.

Worden, J. R., Bowman, K. W., and Jones, D. B.: Two-dimensional characterization of atmospheric profile retrievals from limb sounding observations, J. Quant. Spectrosc. Ra., 86, 45-71, 2004. 